\title{
Assessment of cerebral hemodynamics in multiple sclerosis patients
}

\author{
Nouran M. Salah, Ahmed M. Elsadek, Lobna M. ELnabil, Ayman M. Nassef, Azza A. Abdelaziz and
}

Mohamed A. Abdel Hafeez

\begin{abstract}
Background: Multiple sclerosis (MS) is the most common demyelinating disease of the central nervous system. Autonomic dysfunction is present in a wide range of patients (15-80\%) that might present with abnormalities in the regulation of the cardiovascular system, sleep disturbance, urinary symptoms, sexual problems, and temperature regulation problem. Although the predominant view is that of an autoimmune inflammatory condition, changes in brain vasculature can occur and contribute to pathophysiology.

Objectives: The aim of this study was to evaluate cerebral hemodynamics and assess autonomic dysfunction in patients with MS and explore its relationship with disease status.

Methods: Eighty patients diagnosed with MS according to McDonald criteria 2017 were recruited to this comparative cross-sectional study. All participants underwent assessment of cerebral hemodynamics through transcranial Doppler ultrasonography. Cerebral vasomotor reactivity (CVR) to hypercapnia was measured by the breath-holding index (BHI). They were also assessed for autonomic dysfunction by autonomic questionnaire and blood pressure measurements in both erect and supine positions.
\end{abstract}

Results: BHI values showed decreased CVR and were significantly lower in SPMS than in RRMS patients. Autonomic functions were more affected in SPMS than RRMS with total score $2.58 \pm 1.13$ for the RRMS group and $4.05 \pm 1.04$ for the SPMS group.

Conclusions: Cerebrovascular hemodynamic insufficiency in MS may be secondary to the downstream effects of neuro-inflammatory cascades.

Keywords: Multiple sclerosis, Cerebral hemodynamics, Demyelinating disorders, Transcranial color-coded Doppler, Autonomic dysfunction, Cerebral vasomotor reactivity

\section{Introduction}

Multiple sclerosis (MS) is the most prevalent chronic neurological disorder in young individuals. The disruption of the blood-brain barrier (BBB) is well recognized as a crucial step in the evolution of the multiple sclerosis lesions and is hypothesized to be initiated by auto reactive CD4+ lymphocytes that migrate into the CNS and initiate an inflammatory response. Autonomic disturbance should be considered as one of the crucial components that have an impact on the quality of life

*Correspondence: dr_mohammed_3ly@yahoo.com

Neurology Department, Ain Shams University, Cairo, Egypt outcomes in MS patients [1]. The pathophysiology behind autonomic disturbances remains under dispute, but demyelinating lesions located adjacent to the hypothalamus involving fornix, anterior commissure, internal capsule, and nuclei in the periventricular region of fourth ventricle in the brainstem as well as medullar lesions and optic system might be the basis for autonomic and endocrinal malfunction in MS patients [2]. Sympathetic and parasympathetic dysfunction display different patterns over the course of MS. It has been suggested that parasympathetic dysfunction correlates with disease progression and disability, and its impairment could be

\section{Springer Open}

(- The Author(s). 2021 Open Access This article is licensed under a Creative Commons Attribution 4.0 International License, which permits use, sharing, adaptation, distribution and reproduction in any medium or format, as long as you give appropriate credit to the original author(s) and the source, provide a link to the Creative Commons licence, and indicate if changes were made. The images or other third party material in this article are included in the article's Creative Commons licence, unless indicated otherwise in a credit line to the material. If material is not included in the article's Creative Commons licence and your intended use is not permitted by statutory regulation or exceeds the permitted use, you will need to obtain permission directly from the copyright holder. To view a copy of this licence, visit http://creativecommons.org/licenses/by/4.0/. 
the consequence of MS. Sympathetic dysfunction, on the other hand, is associated with inflammation and clinical activity of MS and may have a pathogenetic role in the development of MS [3]. There was a significant association with increased MS severity, increased score for the pyramidal EDSS functional subscale, and secondary progression, and the association with pyramidal EDSS scale may be due to the proximity of the pyramidal motor pathways to autonomic centers in the brain stem and spinal cord [4]. The aim of this study is to evaluate cerebral hemodynamics and dysautonomia in RRMS and SPMS patients and the relation with disease progression so as not to be underestimated, to improve the quality of life and the possibility of introducing new tools for detection of disease progression as early as possible.

\section{Methods}

We consecutively recruited 80 participants diagnosed as RRMS and SPMS from Multiple Sclerosis outpatient clinics at Neurology Department of Ain Shams University, from 1 August 2018 through 30 July 2020 to this comparative cross-sectional study.

We recruited patients previously diagnosed according to McDonald criteria 2017 with age ranging from 18 to 45 years, patients with relapsing remitting disease course and with secondary progressive disease course. Written informed consent approved by Ain Shams university ethical committee was obtained from the patients participating in the study, or their first-degree relatives if the patient was unable to provide consent due to weakness, after informing them about the study rationale and their right to withdraw from the study at any time without any consequences.

We excluded patients with other comorbidities that may interfere with evaluation of the autonomic nervous system dysfunction as cardiac diseases, alcohol dependence, collagen diseases, diabetes mellitus, renal failure, liver failure, and peripheral neuropathy and patients with past history of cerebrovascular disease, evidence of small vessel ischemic disease, and if there is substantial intracranial pathology in MR imaging. Patients should stop anticholinergic medications $48 \mathrm{~h}$ before testing, whereas sympathomimetics, parasympathomimetic, and beta blockers stopped $24 \mathrm{~h}$ before testing. Patients with primary progressive disease course are not included in this study.

All patients were evaluated by detailed history including personal data, past history, duration of illness, number of relapses, systems affected in each relapse, treatment received during the attack and degree of improvement after recovery and disease-modifying drugs. Full neurological examination and assessment of disability by Extended Disability Status Scale (EDSS) [5] and Multiple Sclerosis Severity Score (MSSS) which relates scores on the Expanded Disability Status Scale (EDSS) to the distribution of disability in patients with comparable disease durations. The MSSS was more powerful than the other methods tested for detecting different rates of disease progression [6].

All patients were studied using a 1.5-T MR scanner (Achieva and Ingenia, Philips medical system, Eindhoven, Netherlands). The protocol includes MR images for the brain (diffusion, flair with sagittal cuts, T1- and T2weighted images) and spinal cord images (cervical and dorsal regions) for assessment of number of lesions, site of lesion (periventricular, juxtacortical, supratentorial, infratentorial), and the presence of brain atrophy.

Intracranial vessels were examined by means of transcranial color-coded Doppler (TCCD) (Esaote My Lab Five Italy), monitoring for middle cerebral arteries (MCA) peak systolic velocity (PSV) during both supine and erect positions 2 min apart using multiphased array low frequency probe $2 \mathrm{MH}$ through the trans temporal window just above the zygomatic arch and in front of the tragus of the ear. The mean flow velocity of MCA in adults ranges from 32 to $82 \mathrm{~cm} / \mathrm{s}$ [7]. The TCCD examination will be repeated using breath holding test to assess cerebrovascular vasomotor reactivity (CVR) to hypercapnia as calculated by means of the breathholding index $(\mathrm{BHI}) . \mathrm{BHI}=\{[\mathrm{MFV}$ at the end of breathholding- resting MFV]/resting MFV $3 \times(100 /$ seconds of breath holding). The value of $1.2 \pm 0.6$ was considered normal, so patients with BHI that is less than 0.6 are considered to have impaired CVR. The breath-holding maneuver was performed according to the procedure of Markus and Harrison: after normal breathing of room air for approximately $4 \mathrm{~min}$, the patients were instructed to hold their breath after a normal inspiration. During the maneuver, the MCA mean blood velocity was recorded continuously. The mean blood velocity at the TCCD display immediately after the end of the breathholding period was registered as the maximal increase of the MCA mean blood velocity (while breath-holding). The time of breath-holding was also registered [8].

Assessment of autonomic functions clinically by measuring systolic and diastolic blood pressure in both supine and erect positions to detect the presence of postural hypotension was done (diagnosis of orthostatic hypotension if there is a drop of $20 \mathrm{~mm}$ of mercury $(\mathrm{mmHg})$ in systolic blood pressure or a drop of 10 $\mathrm{mmHg}$ in diastolic blood pressure within 2 to $5 \mathrm{~min}$ of standing, or if standing causes signs and symptoms) [9].

Questionnaire on clinical autonomic dysfunction was used to assess symptoms of autonomic dysfunction. The scoring from the questionnaire was based on the answer (yes) (score 1) and (no) (score 0) and the total will be calculated for each patient. Higher scores indicate more or worse symptoms [10]. It is composed of six questions assessing different autonomic functions. 
The collected data was revised, coded, tabulated, and introduced to a PC using Statistical Package for Social Science version 25 (SPSS 25) (IBM, San Francisco, CA, USA, 2017). Data was presented and suitable analysis was done according to the type of data obtained for each parameter: mean, standard deviation $( \pm \mathrm{SD})$, and range for parametric numerical data; median and interquartile range (IQR) for non-parametric numerical data; and frequency and percentage for non-numerical data.

Student $T$ test was used to assess the statistical significance of the difference between two study group means. Mann-Whitney test ( $U$ test) was used to assess the statistical significance of the difference of a non-parametric variable between two study groups. ANOVA test was used to assess the statistical significance of the difference between more than two study group means. The Kruskal-Wallis test is used to assess the statistical significance of the difference between more than two study group ordinal variables. Post hoc test is used for comparisons of all possible pairs of group means. Chi-square test was used to examine the relationship between two qualitative variables. Fisher's exact test was used to examine the relationship between two qualitative variables when the expected count is less than 5 in more than $20 \%$ of cells. Correlation analysis (using Spearman's rho and Pearson method) was used to assess the strength of association between two quantitative variables. The correlation coefficient denoted symbolically " $r$ " defines the strength (magnitude) and direction (positive or negative) of the linear relationship between two variables. $r=0-0.19$ is regarded as very weak correlation, $r=0.2-0.39$ as weak correlation, $r=0.40-0.59$ as moderate correlation, $r=0.6-0.79$ as strong correlation, and $r=0.8-1$ as very strong correlation. $P$ value: level of significance, $P>0.05$ : non-significant $(\mathrm{NS}), P<0.05$ : significant $(\mathrm{S}), P<0.01$ : highly significant (HS).

\section{Results}

The sample size was according to this equation: $\mathrm{n}=$ $\frac{2(\mathrm{Za}+\mathrm{Z} 1-\beta) 2 \sigma 2 \text {, }}{\Delta 2}$

where $n$ is the required sample size, and in $Z \alpha, Z$ is a constant (set by convention according to the accepted $\alpha$ error and whether it is a one-sided or two-sided effect).

The total number of patients studied was 80: 13 males (3 diagnosed as RRMS and 10 diagnosed as SPMS) and 67 females (37 diagnosed as RRMS and 30 diagnosed as SPMS) with mean age of $30.4 \pm 6.08$ years for the RRMS group and $45.7 \pm 11.26$ years for SPMS the group. The mean duration of illness was $3(1.25-6)$ years for the RRMS group and $10(8.5-15.5)$ years for the SPMS group. The mean number of relapses was $3(2-5)$ for the RRMS group and $4(3-8.5)$ for the SPMS group. The mean EDSS was $3.43 \pm 1.59$ for the RRMS group and
$6.33 \pm 0.54$ for the SPMS group. There were statistically significant differences between both groups regarding age ( $p$ value $<0.001)$, sex ( $p$ value 0.012 ), duration of illness ( $p$ value $<0.001)$, number of relapses ( $p$ value 0.023 ), and EDSS ( $p$ value $<0.001$ ). As regards diseasemodifying drugs, $42.5 \%$ (17/40) of the RRMS group were on Beta interferons and $47.5 \%$ (19/40) were on fingolimod; meanwhile, $85 \%$ (34/40) of the SPMS group were on rituximab. Multiple Sclerosis Severity Scale (MSSS) was statistically significant between both groups indicating that it is more affected in the SPMS group. The mean value was $6.16 \pm 2.2$ in the RRMS group and 7.61 \pm 1.44 in the SPMS group ( $p$ value $<0.001$ ).

As regards MRI brain findings, high lesion load more than 10 were found in $100 \%(40 / 40)$ of cases of the SPMS group and 70\% (28/40) of cases of the RRMS group; meanwhile, few lesions less than 5 were present in only $12.5 \%(5 / 40)$ of cases of the RRMS group. Brain atrophy was more prominent in the SPMS group representing $45 \%(18 / 40)$ of the cases in comparison with only $5 \%(2 / 40)$ among the RRMS group. In the SPMS group, the lesions were more prominent in the supratentorial region (55\%) (22/40) and infratentorial region (95\%) (38/40); meanwhile, in the RRMS group, the lesions were more prominent in the juxtacortical region (47.5\%) (19/40), the periventricular lesions present in all cases of both groups. There were statistically significant differences between both groups regarding number of lesions ( $p$ value $<0.001)$, site of lesion apart from the periventricular region, and the presence of brain atrophy with $(p$ value $<0.001)$. As regards MRI spinal cord findings, there were no statistically significant differences between both groups regarding number ( $p$ value 0.189 ) and site of lesions ( $p$ value 1.00).

TCCD results showed a statistically significant difference as regards the MCA blood flow monitoring in supine and erect positions between both groups. The mean MCA blood flow in supine position was $84.16 \pm 16.93 \mathrm{~cm} / \mathrm{s}$ for the RRMS group and $65.19 \pm 22.91 \mathrm{~cm} / \mathrm{s}$ for the SPMS group ( $p$ value $<0.001$ ). The mean MCA blood flow in erect position was $80.63 \pm 15.78 \mathrm{~cm} / \mathrm{s}$ for the RRMS group and $58.57 \pm 16.04 \mathrm{~cm} / \mathrm{s}$ for the SPMS group. Chi-square test showed that there is no significant difference between RRMS and SPMS groups in the percentage of decrease of MCA blood flow after changing position as it decreased in $55 \%(22 / 40)$ of patients in the RRMS group and 60\% (24/ 40) of patients in the SPMS group. There is statistically significant difference as regards BHI as it is impaired in $90 \%(36 / 40)$ of cases in SPMS in comparison with only $55 \%(22 / 40)$ of cases in the RRMS group ( $p$ value $<0.001)$ as shown in (Fig. 1).

As regards the autonomic questionnaire, there is statistically significant difference in the autonomic questionnaire total score between both groups with mean 


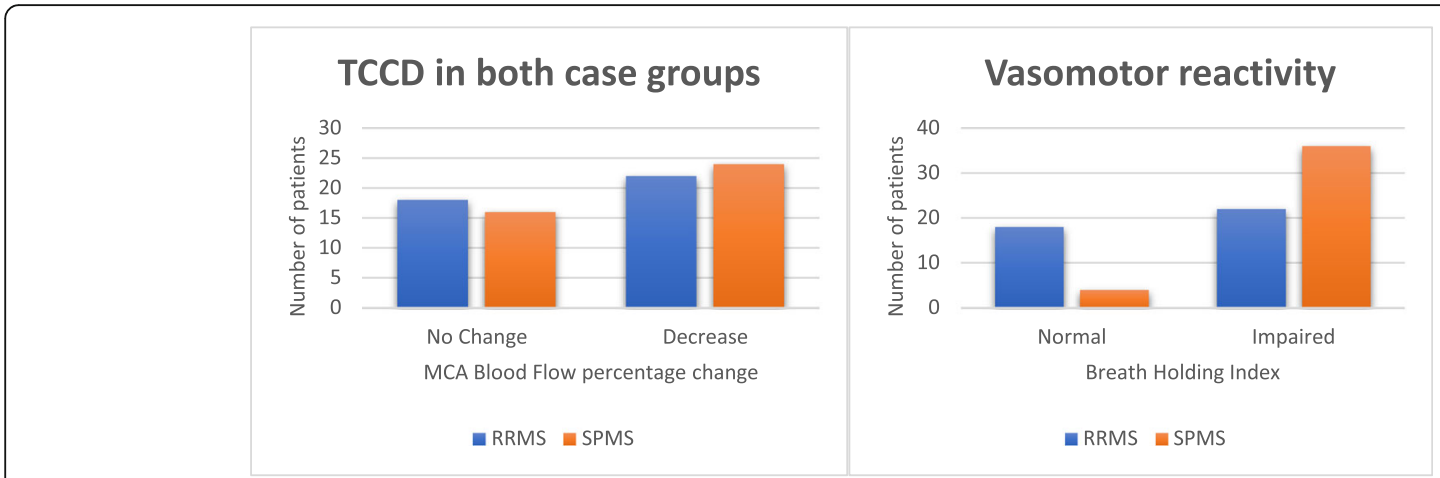

Fig. 1 TCCD assessment between 2 groups of cases as regards MCA blood flow and BHI

score $2.58 \pm 1.13$ for the RRMS group and $4.05 \pm 1.04$ for the SPMS group ( $p$ value $<0.001)$. All the parameters of the questionnaire are statistically significant between both groups apart from postural hypotension, sexual dysfunction, and gastroparesis. Postural hypotension and sphincteric dysfunction are the most affected parameters in the RRMS group with percentage 72.5 (29/40) and 60 (24/40) respectively; meanwhile, color changes and thermal abnormalities in upper and lower extremities are more affected in the SPMS group with percentage 75 (30/40) and $85(34 / 40)$ respectively as shown in Fig. 2.

Blood pressure readings show statistically significant difference between both groups as regards systolic and diastolic readings in the supine position. The mean value of systolic reading was $117.75 \pm 4.8 \mathrm{mmHg}$ in the RRMS group and $123.5 \pm 9.75 \mathrm{mmHg}$ in the SPMS group. Chisquare test showed significant difference between both groups as regards postural hypotension; 35\% (14/40) of the patients in the RRMS group had postural hypotension in comparison with $65 \%(26 / 40)$ of the patients in the SPMS group ( $p$ value 0.007 ). The percentage of decrease of the systolic reading after changing position from supine to erect was statistically significant between both groups as the systolic reading decreased in $57.5 \%(23 / 40)$ of the cases in the RRMS group in comparison with $85 \%(34 / 40)$ of the cases in the SPMS group ( $p$ value 0.007 ). Also, the percentage of decrease of the diastolic reading after changing position was statistically significant between both groups as the diastolic reading decreased in $27.5 \%(11 / 40)$ of the cases in the RRMS group in comparison with $55 \%(22 / 40)$ of the cases in the SPMS group ( $p$ value 0.012 ).

EDSS is directly related to MSSS ( $p$ value $<0.001$ ) in both groups and duration of illness in the RRMS study group ( $p$ value 0.017 ). It is also directly related to the total score of the autonomic questionnaire with $p$ value 0.032 in the RRMS group as shown in Table 1.

MSSS shows direct correlation with the duration of illness $(p$ value $<0.001)$ and percentage of decrease in the MCA blood flow ( $p$ value 0.024 ) in the SPMS group.

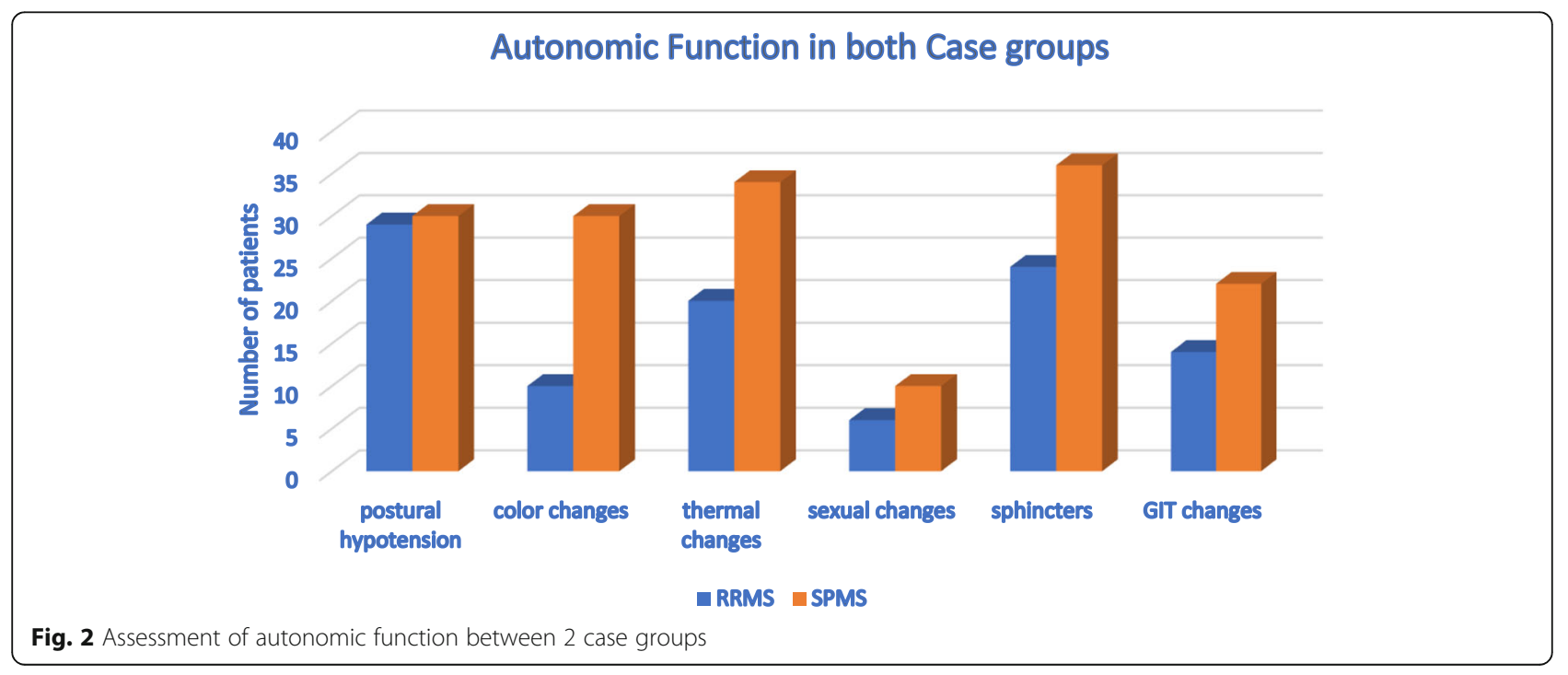


Table 1 Correlation between EDSS and TCCD, autonomic functions, blood pressure, and MSSS in both case groups

\begin{tabular}{|c|c|c|c|c|c|c|}
\hline & \multicolumn{6}{|l|}{ EDSS } \\
\hline & \multicolumn{3}{|l|}{ RRMS } & \multicolumn{3}{|l|}{ SPMS } \\
\hline & Tests used & $p$ value & Significance & Tests used & $p$ value & Significance \\
\hline MSSS & $0.759^{(r)}$ & $<0.001$ & S & $0.582^{(r)}$ & $<0.001$ & $\mathrm{~S}$ \\
\hline Duration of illness (years) & $0.376^{(\mathbf{S})}$ & 0.017 & S & $0.006^{(\mathbf{s})}$ & 0.780 & NS \\
\hline TCCD, \% change & $0.298^{(\mathbf{s})}$ & 0.062 & NS & $0.105^{(\mathrm{s})}$ & 0.465 & NS \\
\hline TCCD, BHI & $-0.197^{(\mathrm{S})}$ & 0.223 & NS & $-0.087^{(\mathbf{S})}$ & 0.928 & NS \\
\hline Autonomic total score & $0.339^{(r)}$ & 0.032 & s & $0.154^{(r)}$ & 0.342 & NS \\
\hline Bl.pr, systolic, \% change & $0.475^{(\mathbf{s})}$ & 0.002 & S & $-0.107^{(\mathbf{s})}$ & 0.846 & NS \\
\hline Bl.pr, diastolic, \% change & $0.054^{(\mathbf{S})}$ & 0.739 & NS & $-0.197^{(\mathbf{S})}$ & 0.385 & NS \\
\hline
\end{tabular}

MSSS Multiple Sclerosis Severity Scale, TCCD transcranial color-coded Doppler, BHI breath holding index, BL.pr blood pressure, RRMS relapsing remitting MS, SPMS secondary progressive MS, S significant, NS non-significant

${ }^{\text {(S) }}$ Spearman's rho test of significance

${ }^{(r)}$ Correlation coefficient

Also, MSSS shows direct relation with the total score of the autonomic questionnaire ( $p$ value 0.021 ) in the RRMS group as shown in Table 2 and 3.

As regards the number of lesions, it is noticed that the percentage of decrease in MCA blood flow upon changing position increased as the number of lesions increased but does not reach statistical significance and also increased with the presence of brain atrophy but does not reach statistical significance in the RRMS group, on the other hand, there is no significant correlation between number of lesions and the occurrence of postural hypotension in the RRMS group.

As regards site of lesions, there is no statistically significant correlation between site of lesions and percentage of decrease in MCA blood flow, impairment of BHI or the total score of the autonomic questionnaire but the prevalence of postural hypotension occurred more with infratentorial lesions (38.46\%) (10/26) followed by periventricular lesions (35\%) (14/40) in the RRMS study group but does not reach statistical significance.

In the SPMS study group, postural hypotension occurred in 65\% (26/40) of the patients who had more than 10 lesions and occurred in $66.67 \%(12 / 18)$ of the patients who had brain atrophy although it does not reach statistical significance. Also, the prevalence of postural hypotension occurred more with cervical lesions (66.67\%) (12/18) followed by periventricular lesions (65\%) (26/40) and infratentorial lesions (63.16\%) (24/38) although it does not reach statistical significance.

\section{Discussion}

We aimed to evaluate cerebral hemodynamics and assess autonomic dysfunction in patients with MS and explore its relationship with disease status.

In our study, we compared blood pressure measurements in supine and erect positions and the incidence of postural hypotension as an indicator of cardiac autonomic dysfunction (CAD). Blood pressure test shows statistically significant difference between both groups as regards systolic and diastolic readings in the supine position. The mean value of systolic reading was $117.75 \pm$ $4.8 \mathrm{mmHg}$ in the RRMS group and $123.5 \pm 9.75 \mathrm{mmHg}$ in the SPMS group. Chi-square test showed significant difference as regards postural hypotension; $35 \%$ of the

Table 2 Correlation between MSSS and TCCD, autonomic functions, and blood pressure in both case groups

\begin{tabular}{|c|c|c|c|c|c|c|}
\hline & \multicolumn{6}{|l|}{ MSSS } \\
\hline & \multicolumn{3}{|l|}{ RRMS } & \multicolumn{3}{|l|}{ SPMS } \\
\hline & Tests used & $p$ value & $\overline{\text { Significance }}$ & Tests used & $p$ value & Significance \\
\hline Duration of illness (years) & $-0.17^{(\mathbf{S})}$ & 0.295 & NS & $-0.769^{(S)}$ & $<0.001$ & $\mathrm{~S}$ \\
\hline TCCD, \% change & $0.271^{(\mathbf{s})}$ & 0.090 & NS & $0.357^{(\mathbf{s})}$ & 0.024 & S \\
\hline $\mathrm{TCCD}, \mathrm{BHI}$ & $-0.144^{(\mathbf{S})}$ & 0.375 & NS & $-0.137^{(\mathbf{S})}$ & 0.398 & NS \\
\hline Autonomic total score & $0.365^{(r)}$ & 0.021 & s & $0.182^{(\mathbf{r})}$ & 0.262 & NS \\
\hline Bl.pr, systolic, \% change & $0.203^{(\mathbf{s})}$ & 0.209 & NS & $0.268^{(\mathbf{S})}$ & 0.094 & NS \\
\hline Bl.pr, diastolic, \% change & $0.073^{(\mathbf{S})}$ & 0.653 & NS & $-0.281^{(\mathbf{S})}$ & 0.079 & NS \\
\hline
\end{tabular}

MSSS Multiple Sclerosis Severity Scale, TCCD transcranial color-coded Doppler, BHI breath holding index, BL.pr blood pressure, RRMS relapsing remitting MS, SPMS secondary progressive MS, S significant, NS non-significant

${ }^{(S)}$ Spearman's rho test of significance

${ }^{(r)}$ Correlation coefficient 
patients in the RRMS group had postural hypotension in comparison with $65 \%$ of the patients in the SPMS group.

These findings were in agreement with previous studies which found that orthostatic symptoms, which are among the symptoms commonly associated with CAD, were reported in $61 \%$ of 104 patients with RRMS in a study from Croatia, and in $24 \%$ of 100 MS patients in a Turkish study. Nevertheless, in both studies, the authors did not distinguish between different forms of orthostatic dysfunction such as dizziness, palpitation, or syncope [11]. This was, however, done in an Iraqi study: in 55 MS patients and 40 age-matched controls, postural dizziness was present in $67 \%$ of patients and $33 \%$ of controls [12]. In a German study (27 RRMS, 13 SPMS), 50\% of the patients reported orthostatic dizziness compared with $17 \%$ of controls [13].

Also, we found that the prevalence of postural hypotension occurred more with infratentorial lesions (38.46\%) followed by periventricular lesions (35\%) in the RRMS study group but does not reach statistical significance; on the other hand, in the SPMS study group, the prevalence of postural hypotension occurred more with cervical lesions $(66.67 \%)$ followed by periventricular lesions (65\%) and infratentorial lesions (63.16\%) although it does not reach statistical significance. This was in agreement with two studies reported significant association between presence of CAD and clinical $(p<0.02)$ and MRI $(p<0.005)$ evidence of brainstem lesions while a Finnish study done on 51 MS patients and 50 controls reported a significant association between the total volume of midbrain MRI lesions and impaired BP response. In contrast, an earlier study from the Netherlands was not able to find a correlation between the number and size of brainstem lesions and abnormalities in autonomic parameters [14].

As regards TCCD findings, in this study, there is statistically significant difference as regards the MCA blood flow monitoring in supine and erect positions between both groups. The percentage of decrease in mean MCA flow upon raising to upright position was more among SPMS patients as it decreased by $-1.9 \%(-12.5$ to $3.7 \%)$ among the RRMS group and $-5.7 \%$ (- 30.1 to $18.3 \%)$ among the SPMS group; however, it did not reach statistical significance. These findings support data by Gonul and colleagues who stated that arterial response to postural variation is a reliable method to assess cerebral autoregulation and may depend on autonomic and neurocardiogenic control systems of cerebral perfusion [15]. These results are consistent with Gonul et al.'s study conducted on 20 RRMS patients and found that the mean cerebral BFV values that were obtained upon raising to the upright position were found to be significantly lower in comparison with the recorded baseline values. The reduction of mean cerebral BFV values after being raised to the upright position was more significant in patients with MS than in control subjects especially in the subgroup with EDSS $>2$. This finding suggests a potential correlation between impaired mean cerebral blood flow responses and MS [15].

In Gonul et al.'s study, they found no relationship between the lesion locations on MRI and the changes of CBFV during head up tilt in patients with MS [15]. The same in our study, there is no statistically significant correlation between site of lesions and percentage of decrease in MCA blood flow, whereas, it is noticed that the percentage of decrease in CBFV upon changing position increased as the number of lesions increased and with the presence of brain atrophy but does not reach statistical significance. The percentage of decrease in MCA blood flow in sitting position shows significant correlation with Multiple Sclerosis Severity Scale (MSSS) and duration of illness in the SPMS group, whereas the correlation between the \% of change in CBFV and EDSS does not reach statistical significance in both groups. This may be explained as the MSSS is more powerful and sensitive than the other methods for detecting disease progression.

As regards assessment of cerebral vasomotor reactivity (CVR) by BHI, in this study, BHI values were 0.64 (0.28-0.95) among the RRMS group and 0.34 (0.290.41 ) among the SPMS group. There is statistically significant difference as it is impaired in $90 \%$ of cases in SPMS in comparison with only $55 \%$ of cases in the RRMS group. These results were in agreement with Lattanzi and colleagues who reported that lower values of MCA were recorded in MS patients than in healthy controls both at rest and at the end of breath holding. BHI values obtained in healthy controls and RRMS and SPMS patients were $1.15 \pm 0.11,0.87 \pm 0.18$, and $0.51 \pm$ 0.20 , respectively. Group-wise, patients showed decreased CVR in comparison to controls and BHI values were significantly lower in SPMS than in RRMS patients [16]. There is no statistically significant correlation between BHI and EDSS, MSSS, the duration of illness, and the number and site of lesions in MRI. In agreement with our findings, another study reported that at linear regression analysis, the disease form was significantly associated with cerebral hemodynamics being the SPMS phenotype a predictor of lower BHI values independently from age, sex, disease duration, EDSS score, and disease-modifying therapy [16]. Another study on a total of 42 patients with MS and 31 healthy controls showed that BHI was lower in patients with MS compared to healthy controls $(0.70 \pm$ 0.43 vs. $0.93 \pm 0.55, P=0.006$ ) documenting a lower cerebrovascular response to hypercapnia. There was no correlation between patient age, EDSS, and disease duration with BHI in patients with MS [17]. 
Table 3 Correlation between duration of illness and TCCD, autonomic functions, and blood pressure in both case groups

\begin{tabular}{|c|c|c|c|c|c|c|}
\hline & \multicolumn{6}{|c|}{ Duration of illness (years) } \\
\hline & \multicolumn{3}{|l|}{ RRMS } & \multicolumn{3}{|l|}{ SPMS } \\
\hline & Spearman's rho & $p$ value & Significance & Spearman's rho & $p$ value & Significance \\
\hline TCCD, \% change & 0.118 & 0.469 & NS & -0.418 & 0.007 & S \\
\hline $\mathrm{TCCD}, \mathrm{BHI}$ & -0.246 & 0.125 & NS & 0.150 & 0.355 & NS \\
\hline Autonomic total score & 0.173 & 0.287 & NS & -0.093 & 0.567 & NS \\
\hline Bl.pr, systolic, \% change & 0.486 & 0.001 & S & -0.443 & 0.004 & S \\
\hline Bl.pr, diastolic, \% change & -0.017 & 0.917 & NS & 0.194 & 0.231 & NS \\
\hline
\end{tabular}

$\overline{T C C D}$ transcranial color-coded Doppler, $B H I$ breath holding index, BL.pr blood pressure, RRMS relapsing remitting MS, SPMS secondary progressive MS, $S$ significant, NS non-significant

As regards assessment of autonomic functions by the questionnaire, there is statistically significant difference in the autonomic questionnaire total score between both groups with mean score $2.58 \pm 1.13$ for the RRMS group and mean score $4.05 \pm 1.04$ for the SPMS group. These results are consistent with a recent study in which there is significantly higher questionnaire total scores in SPMS patients, compared with RRMS [18]. Also, in our study, all the parameters of the questionnaire are statistically significant between both groups apart from postural hypotension, sexual dysfunction, and gastroparesis. Postural hypotension and sphincteric dysfunction are the most affected parameters in the RRMS group with percentage $72.5 \%$ and $60 \%$ respectively; meanwhile, color changes and thermal abnormalities in upper and lower extremities are more affected in the SPMS group with percentage $75 \%$ and $85 \%$ respectively. This observation supports data from other studies reporting slower gastric emptying, bowel dysmotility, constipation, or fecal incontinence as common findings in MS [19].

EDSS is directly related to the total score of the autonomic questionnaire in the RRMS group, but the duration of illness shows no statistically significant correlation with the total score of the autonomic questionnaire. In another study, relative to the effects of disease factors, disease duration showed a weak to moderate correlation with questionnaire total score and moderate correlation between EDSS score and autonomic questionnaire total score $($ Rho $=0.32, p<0.001)[18]$.

This study will open discussion for further research about the underlying vascular disturbance in MS and the disturbance preceding plaque formation aiming at the use of TCCD and other parameters such as MRI perfusion for early detection of disease progression and subsequently prompt treatment measures to improve quality of life.

The main strengths of the current study include the number of recruited participants, which was greater than the sample sizes of other investigations, the analysis of data according to disease stages, and the use of TCCD sonography for real-time CVR assessment. This technique has the advantage to be safe, low-cost, feasible, and easily to perform in clinical practice. Moreover, we studied autonomic dysfunction and its impact on quality of life by the autonomic questionnaire and TCCD as autonomic dysfunction is not uncommon in MS patients and thus non-invasive investigations of autonomic symptoms are warranted to optimize evaluation and disease management.

Our study has some limitations. First, TCD relies on an assumption of constant MCA diameter when interpreting changes in CBFV and this may not be true, especially during head up tilt. Secondly, CBFV is exquisitely sensitive to carbon dioxide levels which may change during head up tilt. Thirdly, correcting the angle of the probe after head up tilt could lead to a different angle insonation of the MCA that could adversely affect the results. Also, A missed statistical method that weighs the sensitivity and specificity of all tests used, as not all patients can do all these tests. Lastly, our study lacks control group as we depend on the standard measurements of MCA blood flow and BHI.

\section{Conclusion}

The main findings of this study were the decrease in CVR observed in patients affected by MS and the relationship between BHI values and disease status, being the progressive phenotype associated with a greater impairment in cerebral hemodynamics than the RR form.

\footnotetext{
Abbreviations

BBB: Blood-brain barrier; BHI: Breath holding index; BFV: Blood flow velocity; CBFV: Cerebral blood flow velocity; CAD: Cardiac autonomic dysfunction; CVR: Cerebral vasomotor reactivity; DMD: Disease-modifying drugs; EDSS: Extended disability status scale; MS: Multiple sclerosis; MSSS: Multiple Sclerosis Severity Score; MCA: Middle cerebral artery; MFV: Mean flow velocity; mmHg: Millimeters of mercury; MRI: Magnetic resonance imaging; PSV: Peak systolic velocity; RRMS: Relapsing remitting multiple sclerosis; SPMS: Secondary progressive multiple sclerosis; SPSS: Statistical Package for Social Science; TCCD: Transcranial color-coded Doppler
}

\section{Acknowledgements}

Not applicable 


\section{Authors' contributions}

A A N., A A N., L M E., A M S., and M A A. conceived of the study and participated in its design and coordination and helped to draft the manuscript. A M S. and N M S. participated in the design of the study and performed the statistical analysis. All authors have read and approved the manuscript.

\section{Funding}

Not applicable

\section{Availability of data and materials}

Dataset is available as master sheet in Excel format and publicly available in Neurology Department, Ain Shams University, through communicating with the corresponding author.

\section{Ethics approval and consent to participate}

The study protocol was approved by Ain Shams University, Faculty of Medicine Research Ethic Committee FWA 000017585 in 2018. Written informed consent was obtained from the patients participating in the study.

\section{Consent for publication}

Not applicable

\section{Competing interests}

The authors declare no competing interests.

Received: 26 November 2020 Accepted: 15 February 2021

Published online: 04 March 2021

\section{References}

1. Koch MW, Metz LM, Agrawal SM, Yong WW. Environmental factors and their regulation of immunity in multiple sclerosis. J Neurol Sci. 2013;324(1-2):106.

2. Rudick RA, Cutter G, Reingold S. The multiple sclerosis functional composite: a new clinical outcome measure for multiple sclerosis trials. Mult Scler. 2002; 8(5):359-65.

3. Shirbani F, Barin E, Lee Y-C, Ng K, Parratt JDE, Butlin M, et al. Characterisation of cardiac autonomic function in MS based on spontaneous changes of heart rate and blood pressure. Mult Scler Relat Disord. 2018:22:120-7.

4. Adamec I, Crnošija L, Junaković A, Krbot Skorić M, Habek M. Progressive multiple sclerosis patients have a higher burden of autonomic dysfunction compared to relapsing remitting phenotype. Clin Neurophysiol. 2018;129: 1588-94.

5. Kurtzke JF. Rating neurologic impairment in multiple sclerosis: an expanded disability status scale (EDSS). Neurology. 1983;33(11):1444-52.

6. Mowry EM, Carey RF, Blasco MR, Pelletier J, Duquette P, Villoslada P, et al. Multiple sclerosis susceptibility genes: associations with relapse severity and recovery. PLoS One. 2013:8:e75416.

7. Yeo LL, Sharma VK. Role of transcranial Doppler ultrasonography in cerebrovascular disease. Recent Pat CNS Drug Discov. 2010:5:1-13.

8. Markus HS, Harrison MJ. Estimation of cerebrovascular reactivity using transcranial Doppler, including the use of breath-holding as the vasodilatory stimulus. Stroke. 1992;23:668-73.

9. Arnold Amy C, Raj Satish R. Orthostatic hypotension: a practical approach to investigation and management. Can J Cardiol. 2017:33(12):1725-8.

10. Suarez GA, Opfer-Gehrking TL, Offord KP, Atkinson EJ, O'Brien PC, Low PA. The autonomic symptom profile: a new instrument to assess autonomic symptoms. Neurology. 1999;52:523-8.

11. Habek M, Crnošija L, Lovrić M, Junaković A, Krbot Skorić M, Adamec I. Sympathetic cardiovascular and sudomotor functions are frequently affected in early multiple sclerosis. Clin Auton Res. 2016;26:385-93.

12. Al-Araji AH, Al-Mahdawi AM, Mohammad Al. Autonomic dysfunction in multiple sclerosis. Neurosciences. 2003;8:177-83.

13. Flachenecker P, Wolf A, Krauser M, Hartung HP, Reiners K. Cardiovascular autonomic dysfunction in multiple sclerosis: correlation with orthostatic intolerance. J Neurol. 1999:246:578-86.

14. Saari A, Tolonen U, Pääkkö E, Suominen K, Pyhtinen J, Sotaniemi K, et al. Cardiovascular autonomic dysfunction correlates with brain MRI lesion load in MS. Clin Neurophysiol. 2004;115:1473-8.
15. Gonul M, Asil T, Balci K, Celik Y, Turgut N, Uzunca I. Changing cerebral blood flow velocity detected by transcranial Doppler ultrasound during head tilt in patients with multiple sclerosis. Eur J Neurol. 2008;15:725-9.

16. Lattanzi S, Acciarri MC, Danni M, Taffi R, Cerqua R, Rocchi C, et al. Cerebral hemodynamics in patients with multiple sclerosis. Mult Scler Relat Disord. 2020;44:102309.

17. Krogias C, Christou I, Tsivgoulis G, Koutroulou I, Schroeder C, Lantinioti C, et al. Functional neurosonology reveals impaired cerebrovascular reactivity in multiple sclerosis. J Neuroimaging. 2019;29(5):589-91.

18. Foschi M, Giannini G, Merli E, Mancinelli L, Zenesini C, Viti B, et al. Frequency and characteristics of dysautonomic symptoms in multiple sclerosis: a crosssectional double-center study with the validated Italian version of the Composite Autonomic Symptom Score-31. Neurol Sci. 2020. https://doi. org/10.1007/s10072-020-04620-1.

19. El-Maghraby TA, Shalby NM, Al-Tawdy MH, Salem SS. Gastric motility dysfunction in patients with multiple sclerosis assessed by gastric emptying scintigraphy. Can J Gastroenterol. 2005;19(3):141-5.

\section{Publisher's Note}

Springer Nature remains neutral with regard to jurisdictional claims in published maps and institutional affiliations.

\section{Submit your manuscript to a SpringerOpen ${ }^{\circ}$ journal and benefit from:}

- Convenient online submission

- Rigorous peer review

- Open access: articles freely available online

High visibility within the field

- Retaining the copyright to your article

Submit your next manuscript at $\boldsymbol{\nabla}$ springeropen.com 